\title{
2. Untersuchung über selektive Absorption im Quecksilberlichtbogen;
} von R. Küch und T. Retschinsky.

(Mitteilung aus dem Laboratorium von W. C. Heraeus.)

(Hierza Taf. IV, Figg. 1 u. 2.)

Wir haben früher ${ }^{1}$ ) aus der Tatsache, daB die WattÖkonomiekurve des bei hohem Druck brennenden Lichtbogens der Quecksilberlampe aus Quarzglas ein Maximum aufweist, geschlossen, daB wir darin die Wirkung regulärer Strahlung zu sehen hätten. Wir konnten im AnschluB daran nachweisen, daB im kontinuierlichen Spektrum des Bogens mit zunehmender Belastung der Lampe die kürzeren Wellenlängen an Intensität schneller zunehmen als die längeren, und daB die Gesamtemission der ultravioletten Strahlung schneller anwachse als die der sichtbaren.

Bei Untersuchung von elf stärkeren Linien des sichtbaren Spektrums in dieser Richtung ergab sich, daB das Ansteigen der Intensität dieser Linien mit zunehmender Belastung in gruppenweise verschiedenem Grade stattfinde, aber in keinem nachweisbaren Zusammenhange mit der Wellenlänge stehe. Für dieses Verhalten bot die Annahme höherer Temperatur des Bogens bei höherer Belastung allein keine Erklärung, und es drängte sich uns daher die Vermutung auf, dab darin eine Wirkung der Absorption des Bogens sich äuBere.

Wir versuchten deshalb zunächst die Absorption einzelner Linien im sichtbaren Spektrum, deren Emissionszunahme mit steigender Belastung wir früher untersucht hatten, zu messen.

$\mathrm{Zu}$ diesem Zweck stellten wir zwei Lampen der zu unseren früheren Untersuchungen ${ }^{2}$ ) verwandten Form hintereinander auf und verglichen für einige Linien des sichtbaren Spektrums die Intensitäten der vorderen Lampe allein $\left(J_{v}\right)$, die der hinteren Lampe allein $\left(J_{h}\right)$, und diejenige beider gleichzeitig brennenden

1) R: Küch u. T. Retschinsky, Ann. d. Phys. 20. p. 563. 1906.

2) I. c. Taf. IV, Fig. 1. 
Lampen $\left(J_{v+h}\right)$ mit den Linien einer dritten konstant brennenden Lampe. $J_{v}+J_{h}-J_{v+h}$ ergab dann die durch die vordere Lampe absorbierte Intensität.

In die Wandung der vorderen, absorbierenden Lampe waren zwei zueinander parallele planparallele Platten eingeschmolzen, um Störungen des Strahlenganges durch die ungleichmäBige Form geblasenen Quarzglases auszuschlieBen. Zwischen beiden Lampen befand sich ein Diaphragma von $2 \mathrm{~mm}$ Breite und $25 \mathrm{~mm}$ Länge; vor der vorderen Lampe wurde ein zweites gleiches Diaphragma aufgestellt. Um es zu vermeiden, daB an den planparallelen Platten sich Quecksilber kondensiere, wenn die Lampe nicht brannte und wir durch sie hindurch die hintere Lampe allein photometrierten, war unterhalb der Lampe ein elektrischer Heizkörper befestigt, der diese Kondensation verhinderte. Einrichtungen zum Höherund Tieferstellen der Lampen ermöglichten die genaue Einstellung des Leuchtfadens. Die Lampen brannten an Akkumulatoren bis anf 1 Proz. konstant, sobald der stationäre Zustand erreicht war, was jedesmal etwa eine halbe Stunde dauerte.

Gemessen wurde mit der früher benutzten Einrichtung; in einem Spektroskop nach Hoffmann (à vision directe) wurden mit Hilfe eines Vergleichsprismas das Spektrum der Vergleichslampe und das zu messende Spektrum übereinander entworfen; zwischen den zu messenden Lichtquellen und dem Spektroskop befand sich ein Nicolpaar zur Schwächung.

Wir hofften anfangs, daB es gelingen würde, für eine gröBere Anzahl von Linien das Verhältnis von Emission und Absorption festzustellen, so daB man hätte untersuchen können, ob dieses Verhältnis für das entsprechende Stück des Spektrums kontinuierlich ineinander ubergehende Werte ergab und eventuell auch ob die Änderung von $E / A$ mit steigender Belastung für benachbarte Wellonlängen nahe gleich sei.

Wir haben diesen Versuchen sehr viel Zeit und Muhe gewidmet, muBten uns aber taberzeugen, dab die uns zu Gebote stehende MeBvorrichtung für den beabsichtigten $Z_{\text {weck }}$ keine genügende Genauigkeit erreichen ließ.

Wir setzen hierher wenigstens eine der Messungsreihen an der gelbgrünen Serienlinie 5461 und der gelben Doppel- 
linie 5769/90, aus der wir sehen, daB bei der gewählten Belastung der Lampen die Absorption einer etwa 1,5 cm dicken Schicht des Lichtbogens für erstere Linie im Mittel 52 Proz., für letztere Linie 15 Proz. des einfallenden Lichtes beträgt. Die Berücksichtigung der in den beobachteten Werten steckenden Werte für die Reflexionen Luft-Quarzglas und QuarzglasQuecksilberdampf erschien bei der Ungenauigkeit der Methode unnötig.

$$
\text { Tabelle I. }
$$

Belastung der hinteren Lampe: 94-96 Volt; 4,2-4,3 Amp. Belastung der vorderen Lampe: 85-95 Volt; 4,2-4,4 Amp.

\begin{tabular}{l|c|c|c|c}
\hline \multicolumn{1}{c|}{$\lambda$} & $J_{v}$ & $J_{v+\lambda}$ & $J_{h}$ & $\begin{array}{c}\text { Absorption } \\
\text { in Proz. des } \\
\text { einfallenden } \\
\text { Lichtes }\end{array}$ \\
\hline \hline $5769 / 90$. & 480 & 737 & 309 & 17 \\
5461 & $47 t$ & 635 & 302 & 48 \\
$5769 / 90$ & 604 & 913 & 375 & 18 \\
5461 & 580 & 780 & 386 & 55 \\
$5769 / 90$ & 558 & 924 & 379 & 4 \\
5461 & 558 & 740 & 353 & 49 \\
$5769 / 90$ & 462 & 740 & 352 & 16 \\
5461 & 484 & 624 & 309 & 55 \\
$5769 / 90$ & 536 & 806 & 345 & 22 \\
5461 & 511 & 650 & 302 & 54
\end{tabular}

Aus dieser Messungsreihe geht hervor, daB die Linie 5461 und das Paar 5769/90 im Lichtbogen in sehr verschieden hohem Grade absorbiert werden. Wir durften annehmen, daB dieses auch für die übrigen Linien der Fall sein muBte, und konnten infolgedessen voraussehen, $\mathrm{daB}$ die verschiedene $\mathrm{Ab}$ sorption der verschiedenen Linien sich dann geltend machen würde, wenn wir das Intensitätsverhältnis der Linien im Spektrum verschieden dicker Schichten des Lichtbogens miteinander verglichen. ${ }^{1}$ ) Glichen wir die absoluten Intensitäten einer dünnen und einer dicken strahlenden Schicht so gegeneinander ab,

1) Vgl. hierzu H. Kayser, Handbuch der Spektroskopie 2. p. 232 und p. $241 \mathrm{ff} .1902$. 
daB irgend eine Linie in dem Spektrum beider Schichten gleiche Intensität besaB, so muBten alle Linien, welche stärker absorbiert werden als die gleichgestellten, im Spektrum der dünnen Schicht gröBere Intensität zeigen als in demjenigen der dicken Schicht, und alle Linien, welche schwächer absorbiert werden, umgekehrt sich verhalten.

Zur experimentellen Prïfung dieser SchluBfolgerung wählten wir folgende Versuchsanordnung.

Wir stellten uns zwei vollkommen gleiche Quecksilberlampen von folgender Form her:

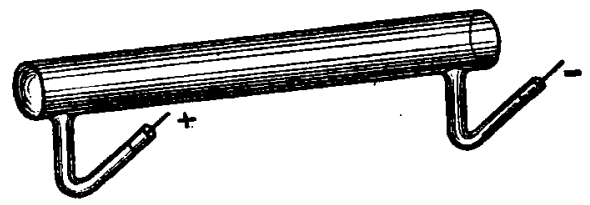

Ein ca. $20 \mathrm{~mm}$ weites und ca. $460 \mathrm{~mm}$ langes Rohr ist an beiden Enden mit eingeschmolzenen, planparallelen Platten verschlossen. Nahe den Rohrenden sind zwei ca. $50 \mathrm{~mm}$ lange Rohrstücke senkrecht zur Achse des weiten Rohres angesetzt, von denen eines ca. $15 \mathrm{~mm}$, das andere ca. $10 \mathrm{~mm}$ weit ist, und von denen das weitere den positiven und das engere den negativen Quecksilberpol aufnimmt. Die Stromzuführung geschieht durch Nickelstahlstifte, die in Rohrstutzen eingeschliffen sind, welche vom unteren Ende der Polrohre schräg nach oben führen. Die Abdichtung der Nickelstahlstifte geschieht durch einen QuecksilberverschluB, der durch Chattertonkitt überdeckt wird. Die PolgefäBe werden in Gefäße aus Metall eingesetzt, durch welche Kühlwasser flieBt.

Durch Regulierung der Kühlung und der elektrischen Belastung ist es möglich, beide Lampen bei jeder beliebigen Stromstärke und Spannung dauernd absolut konstant brennen zu lassen, so daB es erlaubt war, das Spektrum der Querdurchsicht der einen Lampe mit dem Spektrum der Längsdurchsicht der anderen Lampe zu vergleichen, wenn beide mit genau der gleichen Charakteristik brannten.

Wir hatten zuerst versucht, die Längsdurchsicht mit dem Spiegelbilde der Querdurchsicht derselben Lampe zu vergleichen, muBten hiervon aber absehen, da die selektive $R e$. 
flexion des verwandten Silberspiegels das Resultat im Ultraviolett vollkommen fälschte. Von einem Drehen derselben Lampe dem Spektrographen gegenüber aus der Stellung der Querdurchsicht in diejenige der Längsdurchsicht sahen wir ab, da die jedesmalige genaue Orientierung der letzteren Stellung außerordentlich zeitraubend gewesen wäre.

Diese Orientierung muBte sehr genau geschehen, da wir den Spektrographen in grober Entfernung von der Lichtquelle aufstellen muBten. Sollten nämlich die von den hinteren Teilen des Rohres kommenden Strahlen den von den vorderen Teilen kommenden gegenüber nicht schon durch die größere Entfernung geschwächt zur Wirkung kommen, so mußte die Entfernung des Spektrographen von der Lampe so gro $\beta$ gewählt werden, daß ihr gegenüber die Länge des Rohres vernachlässigt werden konnte. Wir haben den Spektrographen deshalb in einer Entfernung von ca. $15 \mathrm{~m}$ von den Lampen aufgestellt.

Die beiden Lampen wurden in einer Entfernung von etwa $1,5 \mathrm{~m}$ voneinander mit den zugehörigen Kühlvorrichtungen auf einem Tische so aufgestellt, daB die eine ihre Breitseite, die andere eine Stirnfläche dem Spektrographen zukehrte.

Der Spektrograph von Schmidt \& $\mathrm{Haensch}^{1}$ ), mit Glasund Quarzoptik, war auf einer Drehscheibe so aufgestellt, daB die Kollimatorachse abwechselnd auf die-Längs- und die Querlampe gerichtet werden konnte. Die genaue Orientierung der Längslampe geschah so, daB die Achse eines Fernrohres, welches zwischen Lampe und Spektrograph, etwa 1,5 m von letzterem entfernt, aufgestellt war, horizontal und in die Verlängerung der Kollimatorachse gerichtet wurde. Mit diesem Fernrohr wurde ein hinter der Längslampe gelegener Punkt fixiert, und dann die Lampe so aufgestellt, daB dieser Punkt in zentraler Lage durch die beiden Stirnflächen der Lampe hindurch sichtbar war. In entsprechender Weise geschah die Aufstellung der Querlampe. Die Drehscheibe, auf welcher der Spektrograph stand, hatte zwei Anschläge, welche die Richtungen der Kollimatorachse auf die Quer-und die Längslampe fixierten. Lampen und Spektrograph befanden sich in zwei durch eine Tür getrennten Räumen; verschlieBbare Diaphragmen

1) F. Schmidt \& Haensch, Katalog II. p. 38. September 1903. 
in der Tür erlaubten es, das Licht jeder Lampe gesondert zum Spektrographen zu senden, auch wenn beide Lampen gleichzeitig brannten. Diffuses Licht wurde durch geeignete Anordnung mehrerer Diaphragmen abgehalten. Die Intensität des von jeder Lampe zum Spektrographen gelangenden Lichtes konnte durch variierbare Diaphragmen geändert werden.

Es stellte sich nun alsbald heraus, daB in der Tat die Intensitätsverteilung im Spektrum der Längsdurchsicht eine so wesentlich andere war, als in demjenigen der Querdurchsicht, daB der Unterschied schon dem bloßen Auge sichtbar wurde. Die Gesamtfarbe des Lichtes der Längsdurchsicht erscheint bei weitem gelber und röter als diejenige des Lichtes der Querdurchsicht.

Wir verglichen nunmehr zunächst das sichtbare Spektrum der Querdurchsicht mit demjenigen der Längsdurchsicht durch okulare Beobachtung, indem wir durch einmalige Spiegelung des Lichtes der Querlampe und Vergleichsprisma die beiden Spektren übereinander entwarfen.

Es gelingt leicht, durch ein veränderbares Diaphragma, welches vor die Querlampe gestellt wird, die Intensitäten beider Spektren so gegeneinander abzugleichen, daB irgend eine Linie die gleiche Intensität zeigt. Wählt man hierzu z. B. die grüne Serienlinie 5461, so sieht man ohne weiteres, daB das gelbe Paar 5769/90 im Spektrum der Längslampe sehr erheblich gröBere Intensität besitzt. Annähernd die gleiche Intensität in beiden Spektren zeigt die blaue und violette Serienlinie (4358 und 4047). Alle übrigen Linien sind im Längsspektrum stärker; ebenso das kontinuierliche Spektrum. Stellt man durch Veränderung des Diaphragmas der Querlampe z. B. das gelbe Paar 5769/90 in beiden Spektren gleich, so erscheinen die Serienlinien 5461, 4358, 4047 in der Querlampe stärker, andere Linien sind in der Längslampe stärker. Betrachtet man benachbarte Linien von verschiedener Emission, so findet man, daß beim Gleichstellen der stärkeren Linie die schwächere allemal in der Längslampe stärker ist. Besonders klar tritt dieses Verhältnis bei den nahe beieinander liegenden blauen Linien 4358, 4347, 4339 hervor, indem bei gleichgestellter Linie 4358 die Linien 4347 und 4339 ganz auBerordentlich in der Längslampe überwiegen. Auch die 
schwächeren Linien im Gelbgrün und Grün bilden zahlreiche Beispiele.

Da wir voraussetzen dürfen, daB diejenige von zwei Linien in der dickeren Schicht der dünneren gegenüber am meisten absorbiert wird, welche am wenigsten an Intensität gewinnt, so folgt aus den mitgeteilten Beobachtungen, da $\beta$ von nahe beieinander liegenden Linien im Quecksilberlichtbogen die stärkere am meisten absorbiert wird. ${ }^{1}$ )

Da wir die erhaltenen Resultate insbesondere auch im Ultraviolett prüfen wollten, wo die zahlreichen Serienlinien besonderes Interesse boten, so gingen wir dazu über, auf photographischem Wege zunächst im sichtbaren Gebiet die beobachteten Tatsachen zu fixieren und wandten hierfür die folgende Methode an:

Wie oben erwähnt, konnte der Spektrograph mittels einer Drehscheibe abwechselnd auf die Längs- und die Querlampe gerichtet werden. Wir ermittelten nun durch Veränderung des Diaphragmas der Querlampe zunächst ein Intensitäts-

1) Auf Veranlassung von Hrn. Prof. J. Stark stellten wir nach AbschluB der vorliegenden Untersuchung an einigen stärkeren Linien auf spektralphotometrischem Wege für die Querlampe und für die Längslampe das Intensitätsverhăltnis zu den Linien einer Vergleichslampe fest. Wir bedienten uns der früher (Ann. d. Phys. 20. p. 563. 1906) benutzten Anordnung. Setzen wir die Intensitäten aller Linien der Vergleichslampe und der Linie 5461 in Quer- und Längslampe gleich 100, so ergeben sich für die Intensitäten der anderen Linien die in der Tabelle zusammengestellten Zahlen.

Tabelle II.

\begin{tabular}{l|c|c|c}
\hline \multicolumn{1}{c|}{$\lambda$} & Quer & $\begin{array}{c}\text { Vergleichs- } \\
\text { lampe }\end{array}$ & Llings \\
\hline $5790 / 69$ & 81 & 100 & 212 \\
5461 & 100 & 100 & 100 \\
4959 & 66 & 100 & 443 \\
4916 & 71 & 100 & 443 \\
4358 & 92 & 100 & 96 \\
$4347 / 39$ & 64 & 100 & 463
\end{tabular}

Das Verbăltnis der Intensităt in Quer- und Lăngslampe für die Linie 4358 ist dem der Linie 5461 gleich; für die anderen Linien ergibt sich ein starkes Anwachsen der relativen Intensitäten in der Längslampe. 
verhältnis, bei welchem bei genau gleicher Expositionszeit geeignete Linien in der Querlampe deutlich stärker erschienen als in der Längslampe. Nun exponierten wir Längs- und Querlampe abwechselnd jedesmal genau gleich lang mit drei verschiedenen passend gewählten Expositionszeiten.

Es zeigte sich nämlich, dab man, um bei allen Linien die Intensitätsunterschiede erkennen zu können, mit einer einzigen Aufnahme jedes Spektrums nicht auskommt. Die Stärke zweier Linien läBt sich, wenn es sich um geringe Unterschiede handelt, nur vergleichen, wenn eine gewisse Schwärze der Platte noch nicht überschritten ist. Man braucht zum Vergleich der stärkeren Linien deshalb kürzere Expositionszeiten, bei denen die schwächeren Linien noch nicht herauskommen. Die Belastung beider Lampen wurde bei allen Aufnahmen untereinander genau gleich gehalten. Die kurz exponierten Aufnahmen erlauben nun den Vergleich der starken, dio länger exponierten den der schwächeren und schwächsten Linien des Spektrums.

Fur die Photographie des sichtbaren Spektrums verwandten wir mit "Orthochrom T" sensibilisierte Platten der Farbwerke Höchst, für die Photographie des Ultravioletts gewöhnliche Schleussnerplatten des Handels. Die Reproduktion der Aufnahmen ist in etwa doppelter Vergrößerung des Originals geschehen.

Wir haben eine sehr groBe Anzahl ron Aufnahmen gemacht, müssen uns aber naturgemäB darauf beschränken, die gefundenen GesetzmäBigkeiten an einem typischen Beispiel, das sich auf Taf. IV abgebildet findet, zu demonstrieren.

I. Bichtbares Bpektrum (Taf. IV, Fig. 1).

Belastung beider Lampen 5 Amp., 142 Volt. Spektrum 1, 3, 5: Längslampe; Spektrum 2, 4, 6: Querlampe. Spektrum 1 und 2: Expositionszeit 1 Stunde; Spektrum 3 und 4: Expositionszeit 2 Min.; Spektrum 5 und 6: Expositionszeit 5 Sek.

Die Intensitäten beider Lampen sind durch Diaphragmen so abgeglichen, daB die stärksten Linien 5461-4358-4047 in Querlampe deutlich stärker sind, als in Längslampe. Es ergibt sich dann, daß sämtliche übrigen Linien in der Längslampe stärker sind, als in der Querlampe. Auch das konti- 
nuierliche Spektrum ist in der Längslampe stärker als in der Querlampe. Im einzelnen läBt sich dann noch vielfach erkennen, daB ron benachbarten Linien die schwächeren in der Längslampe mehr überwiegen als die stärkeren.

II. Ultraviolettes Bpoktrum (Taf. IV, Fig. 2).

Belastung beider Lampen 5,1 Amp., 145 Volt. Spektrum 1, 3, 5: Längslampe; Spektrum 2, 4, 6: Querlampe. Spektrum 1 und 2: Expositionszeit 6 Min.; Spektrum 3 und 4: Expositionszeit 30 Sek.; Spektrum 5 und 6: Expositionszeit 5 Sek.

Die Intensitäten beider Lampen sind durch Diaphragmen so abgeglichen, daß die Linien 4359 (blau) und 4047 (violett), sowie noch einige andere Serienlinien, von denen weiter unten ausfübrlicher die Rede ist, in der Querlampe stärker sind als in der Längslampe. Es ergibt sich dann zunächst, daB die zwischen 4047 und 3341 liegenden schwächeren Linien in der Lüngslampe stärker sind als in der Querlampe. Wir haben also auch hier das Resultat, $\mathrm{daB}$ von benachbarten Linien die schwächeren in der Längslampe mehr überwiegen als die stärkeren.

Es lag nun nahe, die im Ultraviolett des Quecksilberspektrums vorhandenen Serienlinien bezüglich ihrer Absorption etwas genauer zu vergleichen und es ergab sich in der Tat, daB hier eine deutliche GesetzmäBigkeit vorliegt.

Im Quecksilberspektrum sind bekanntlich sechs Serien (zwei Nebenserien von Triplets) rorhanden. In der folgenden Tabelle haben wir das Resultat unserer Beobachtungen an den Linien dieser sechs Serien zusammengestellt. Die Beobachtungen geschahen an den Originalplatten mit dem Mikroskop. In den VergröBerungen sind die Intensitätsverhältnisse in den extremeren Fällen gleichfalls sichtbar.

Tabelle III.

Erste Nebenserie.

\begin{tabular}{|c|c|c|c|c|c|c|c|c|c|}
\hline$n$ & $\lambda$ & Quer & Lüngs & 2 & Quer & Längs & $\lambda$ & Quer & Lakngs \\
\hline 4 & $\mathbf{3 6 5 0}$ & \multicolumn{2}{|c|}{ gleich } & 3125 & st\&rker & & 2967 & stärker & \\
\hline 5 & 3021 & \multicolumn{2}{|c|}{ gleich } & 2652 & stărker & & 2534 & \multicolumn{2}{|c|}{ gleich } \\
\hline 6 & 2803 & & stärker & 2482 & gle & ich & 2378 & & stärker \\
\hline 7 & 2699 & & stälrker & 2399 & & stärker & 2301 & & starker \\
\hline
\end{tabular}


Tabelle III (Fortsetzung).

Zweite Nebenserie.

\begin{tabular}{c||c|c|c||c|c|c||c|c|c}
\hline$n$ & $\lambda$ & Quer & Lxngs & $\lambda$ & Quer & Längs & $\lambda$ & Quer & Längs \\
\hline 3 & 5461 & - & - & 4358 & stärker & & 4047 & stărker & \\
4 & 3841 & stärker & & 2893 & stärker & & 2752 & stärker & \\
5 & 2925 & & stärker & 2576 & & stärker & 2464 & & stärker \\
6 & 2759 & & stärker & 2446 & & stärker & 2345 & & stärker \\
7 & 2675 & & stärker & & & & & &
\end{tabular}

Die ersten, zweiten und dritten Linien der Triplets stehen je untereinander. Für jede Linie ist angegeben, ob sie in Querlampe oder in Längslampe stärker oder in beiden gleich ist. Es ergibt sich das Resultat, das allgemein in den Serièn die Linien gröBerer Wellenlängen entweder in Querlampe stärker oder in beiden Lampen gleich, daB die Linien kleinerer Wellenlängen in der Längslampe stärker sind; d. h. also: in allen Serien werden die Linien kleiner Wellenlängen weniger absorbiert als diejenigen großer Wellenlängen.

Die Triplets der ersten Nebenserie zeigen bekanntlich einen zusammengesetzten Bau, indem in jedem Triplet die erste Linie aus vier, die zweite aus drei, die dritte aus zwei Komponenten besteht. Aus unseren Versuchen ergibt sich, daB in der ersten und zweiten zusammengesetzten Linie jeden Triplets die stärkste Komponente, die auch zugleich diejenige kleinster Wellenlänge ist, stärker absorbiert wird als ihre Begleiter; die relativen Intensitäten der schwächeren Komponenten sind in der Längslampe gröBer als in der Querlampe. ${ }^{1}$ ) Die allgemeine Regel, dab von benachbarten Linien die stärkeren

1) In dem Spektrum unserer Längslampe erscheint infolge der großen Schichtdicke auber den von Eder und Valenta gemessenen Linien 2806/4/3-2483/2/2 noch die Linie 2805,3. Wir haben sie mittels einer Skala unter dem Mikroskop bis auf 0,2 $\AA$.-E. genau interpoliert. Die Rechnung ergibt, daB diese Linie, wie auch die Linien 2806/4/3 und 2483/2/2 dem Triplet der ersten Nebenserie für $n=6$ angehört. Sein Bau wird dadurch dem der ersten zwei Triplets analog. Die Differenzen zwischen den Schwingungszahlen der Komponenten der ersten Linie dieses Triplets sind: $15,6-11,4-9,0$; der zweiten Linie: 16,2-12,3. 
Linien stärker absorbiert werden als die schwächeren, gilt also auch für die Komponenten dieser zusammengesetzten Serienlinien. ${ }^{1}$ )

Das Quecksilberspektrum hat bei 2536,7 bekanntlich noch eine besonders starke Linie, die nicht zu den Serienlinien gehört. Über die Absorptionsverbältnisse dieser Linie erlauben uns unsere Spektrogramme keinen sicheren SchluB zu ziehen, da die Linie uns sehr oft umgekehrt und an ihrer Stelle ein bis zu $5 \AA$.-E. breiter weißer Strich erschien. Die Linie erschien nur bei sehr niedriger Belastung nicht umgekehrt.

Die bisherigen Untersuchungen haben uns das allgemeine Resultat ergehen, daB die Intensitätsverteilung im Linienspektrum des Quecksilberbogens bei konstanter Temperatur eine sehr verschiedene ist, je nachdem das Spektrum von einer dünnen oder von einer dicken Schicht ausgestrahlt wird. Früher ${ }^{2}$ ) haben wir nachweisen können, daB mit steigender elektrischer Belastung der Quecksilberlampe, bei welcher die mittlere Temperatur ${ }^{3}$ ) und der Dampfdruck steigt, gleichfalls eine Änderung der Intensitätsverteilung eintritt. Wir ver-

1) Wir lieBen durch die Lampen, welche uns den Lichtbogen geliefert hatten, die Entladungen eines kleinen Induktoriums von etwa $2 \mathrm{~cm}$ Funkenlänge gehen, dessen Primärspule mit zwei Akkumulatoren gespeist wurde, indem wir zur Erzielung gröBerer Intensität Rohr und Elektroden etwas erhitzten, und photographierten die Spektren der Langsund der Querdurchsicht in der gleichen Weise, wie es oben beschrieben wurde. Das Resultat war genau das gleiche, wie beim Bogen. Ganz besonders deutlich war dies an den zussmmengesetzten Linien der ersten Nebenserie zu erkennen. Die Intensitătsuntersehiede der einzelnen Komponenten untereinander waren in der Querlampe bei weitem stärker, als in der Längslampe.

Nach AbschluB des Manuskriptes erschien die Notiz von R.W. Wood in Heft 25, 1906 der Physik. Zeitschr. p. 926, der den Unterschied in der Farbe einer dünnen und einer dicken Schicht elektrisch leuchtenden Wasserstoffs bemerkt und als wahrscheinlichen Grund die stärkere $\mathbf{A b}$ sorption der Linie $H_{a}$ gegenüber $H_{\beta}$ und $H_{\gamma}$ angibt. Aus der beigegebenen Skizze scheint hervorzugehen, daB Wood aus geringer Entfernung beobachtete. Wie oben bemerkt, muB man die Erscheinung deutlicher in gröBerem Abstand erbalten.

2) l. c. p. 578 .

3) R. Kü ch u. T. Retschinsky, Ann. d. Phys. 22. p. 595.1907. 
glichen nunmehr das Spektrum der hochbelasteten Lampe in Querdurchsicht mit dem Längsspektrum der niedrig belasteten Lampe. Es fand sich, daB durch Steigerung der Belastung das Querspektrum dem Längsspektrum der niedrig belastet bleibenden Lampe genähert werden kann. Die Intensität aller schwächeren Linien steigt mit steigender Belastung schneller an, als die der stärkeren, so daB also Steigerung der Belastung in demselben Sinne wirkt, wie VergröBerung der Schichtdicke. Nun haben wir friher ${ }^{1}$ ) mitgeteilt, daB der Lichtbogen unserer Lampe mit steigender Belastung sich einschnürt. Wir nehmen daher an, daB mit dieser Einschnürung infolge gröBerer Dichte der leuchtenden Schicht größere absorbierende Wirkung der letzteren eintritt, und $\mathrm{daB}$ in der früher beobachteten Änderung der Intensitätsverteilung im Spektrum mit steigender Belastung neben der Wirkung erhöhter Temperatur vor allem auch die Wirkung erhöhter Absorption in die Erscheinung trat.

Betrachtet man von diesem Standpunkt aus jetzt die WattÖkonomiekurve der hochbelasteten Quecksilberlampe \%), so erkennt man in dem Abfall der Kurve nach Erreichung des Maximums die Wirkung höherer Temperatur und kann den Umstand, daB die Kurve nach anfänglichem steilem Abfall abflacht, so deuten, daB in dem steilen Ast der Kurve die Absorption der gelben Doppellinie 5790/69, von deren Emission neben derjenigen der grüngelben Serienlinie 5461 - die Gesamtökonomie in erster Linie abhängt, noch gering ist, während in der Abflachung sich die mit der Einschnürung des Leucht. fadens gröBer werdende Absorption dieses Linienpaares ausdrückt.

In welcher Weise erhöhte Temperatur für sich allein das Spektrum des Quecksilberbogens verändert, konnten wir nur erkennen, wenn wir zwei Schichten von gleicher absorbierender Wirkung und verschiedener Temperatur verglichen. Wir konnten gleiche absorbierende Wirkung zweier Schichten nach den vorher mitgeteilten Ergebnissen annehmen, wenn ein eng begrenztes Spektralgebiet in beiden Spektren gleiche relative Intensität aller Linien zeigte.

1) 1. c. p. 566, Taf. IV, Fig. 3.

2) l. c. p. 569 . 
Wie oben erwähnt, gelingt es nun zwar durch böhere Belastung unserer Lampe das Spektrum dem Spektrum der Längsdurchsicht der niedrig belasteten Lampe in diesem Sinne nahe gleich zu machen. Es ergab sich indessen, dab die mit steigender Belastung eintretende Verbreiterung aller Linien einen genaueren Vergleich mit den unverbreiterten Linien der Längsdurchsicht unmöglich machte.

Wir können indessen eine höhere Belastung unserer Lampe in doppelter Weise vornehmen. Entweder lassen wir die Kühlung der Elektroden dieselbe bleiben, dann steigt mit höherer Belastung der Dampfdruck und die Elektrodenspannung, während die Stromstärke nach Erreichung des stationären Zustandes dieselbe ist; von dieser Steigerung der Belastung war bisher die Rede. Oder wir verstärken die Kühlung der Elektroden, dann steigt mit höherer Belastung die Stromstärke, während Dampfdruck und Spannung dieselben bleiben. Für beide Fälle haben wir Steigerung der mittleren Temperatur und Sinken des spezifischen Wattverbrauches nachgewiesen.

Es stellte sich heraus, dab nur im ersteren Falle Verbreiterung der Linien eintritt, so dab diese Verbreiterung in unserer Lampe als eine Folge des erhöhten Druckes nachgewiesen erscheint.

Der Druck in unserer Lampe wird nun annähernd gemessen durch den Spannungsabfall pro Zentimeter Lichtsäulenlänge, wenn man etwa 12 Volt für Elektrodengefälle in Abzug bringt. Zwei Lampen mit gleichem Spannungsabfall pro Zentimeter Lichtsäulenlänge haben demnach annähernd den gleichen Dampfdruck und infolgedessen gleich breite Linien.

Von diesen Überlegungen geleitet, gelang es uns zwei vergleichbare Schichten von gleicher absorbierender Wirkung in dem Sinne, da $B$ in eng begrenzten Spektralgebieten die Intensitätsverteilung die gleiche war, herzustellen, die verschiedene Temperatur besaBen. Wir fertigten eine Lampe an, welche genau so konstruiert war wie die beiden $46 \mathrm{~cm}$ langen Lampen, deren Leuchtrohr indessen nur etwa $10 \mathrm{~cm}$ lang war. Diese und eine der $46 \mathrm{~cm}$ langen Lampen wurden für Längsdurchsicht montiert. Beide Lampen wurden mit etwa dem gleichen Spannungsabfall pro Zentimeter Lichtsäule betrieben. Der langen Lampe gaben wir kleine, der kurzen Lampe große 
Stromstärke. Das Spektrum der kurzen Lampe muBte deshalb ein solches höherer Temperatur sein.

Wenn wir die lange Lampe mit 125 Volt und 2,2 Amp., die kurze Lampe mit 41 Volt und 10,4 Amp. belasteten, zeigten beide Längsspektren für kleine Spektralbezirke die gleiche Intensitätsverteilung. Wir konnten z. B. durch Abgleichung mittels Diaphragmen erreichen, daB im Ultraviolett zwischen 3908 und 3341 die starken wie die schwachen Linien in beiden Spektren etwa gleich waren.

Wir stellten nun mittels Diaphragmen die Intensitäten beider Lampen so ein, daB die Linien 4358-4109-4078-4.047 in der langen Lampe, $d . h$. derjenigen niederer Temperatur, stärker waren als in der hurzen Lampe.

Es ergab sich das Resultat, daB unter diesen Umständen die Linien ron etwa 3908 bis 2759 in beiden Lampen annähernd gleich, die Linien von da ab bis zum Ende des Spektrums in der kurzen Lampe stärker waren.

Bei gleicher absorbierender Wirkung unterscheidet sich also das Spektrum höherer Temperatur von demjenigen niedrigerer Temperatur dadurch, daß die Linien kürzerer Wellenlängen relativ größere Intensität besitzen.

\section{Schlußfolgerungen.}

Wir haben nachgewiesen, daB verschieden dicke Schichten des Quecksilberbogens von derselben Temperatur verschiedene Intensitätsverteilung im Linienspektrum zeigen. Im Spektrum der dickeren Schicht sind die schwächeren von benachbarten Linien relativ stärker. Dies ist als eine Wirkung der Absorption im Bogen, die wir direkt an zwei Linien gemessen haben, zu deuten, so daB wir folgern können: Im Quecksilberbogen wird von benachbarten Linien die Linie gröBerer Emission auch stärker absorbiert. Wir haben früher wahrscheinlich gemacht, daB reguläre Strahlung im Bogen unserer Lampe eine Rolle spielt. Bringen wir dementsprechend die Folgerungen aus dem Kirch hoffschen Satze zur Anwendung, so müssen wir, da $E / A=e^{1}$ ), und da $e$ für benachbarte Wellenlängen bei derselben Temperatur nur wenig verschiedene Werte annehmen

1) Emission des schwarzen Körpers.

Annalen der Phyilk. IV. Folge. 22. 
866 R. Küch u. T. Retschinshy. Selektive Absorption etc.

kann, verlangen, da $B$ größerem $E$ auch größeres $A$ entspricht. Dies ist im Quecksilberbogen tatsächlich der Fall.

Wir fanden ferner, daB bei gleicher absorbierender Wirkung der Schichten die Spektren von Schichten verschiedener Temperatur sich dadurch unterscheiden, daB bei höherer Temperatur die Linien kleiner Wellenlängen relativ stärker sind, als bei niedriger. Auch dies Verhalten entspricht einer Folgerung aus dem Kirchhoff schen Satze. $e$ wächst mit steigender Temperatur schneller für die kurzen Wellenlängen. Da $E / A=e$, so muB bei gleichem $A$ auch $E$ für die kurzen Wellen mit steigender Temperatur schneller wachsen als für die langen. Hanau, Dezember 1906.

(Eingegangen 31. Januar 1907.) 


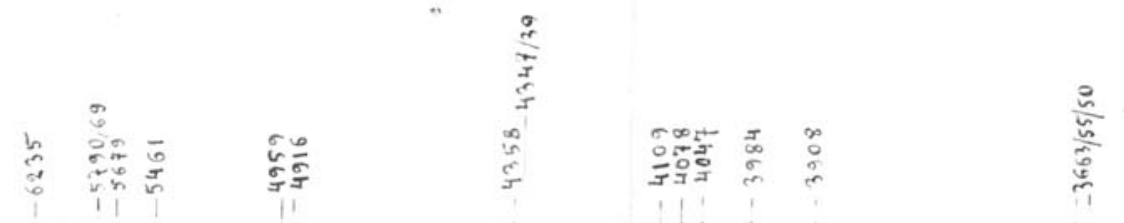

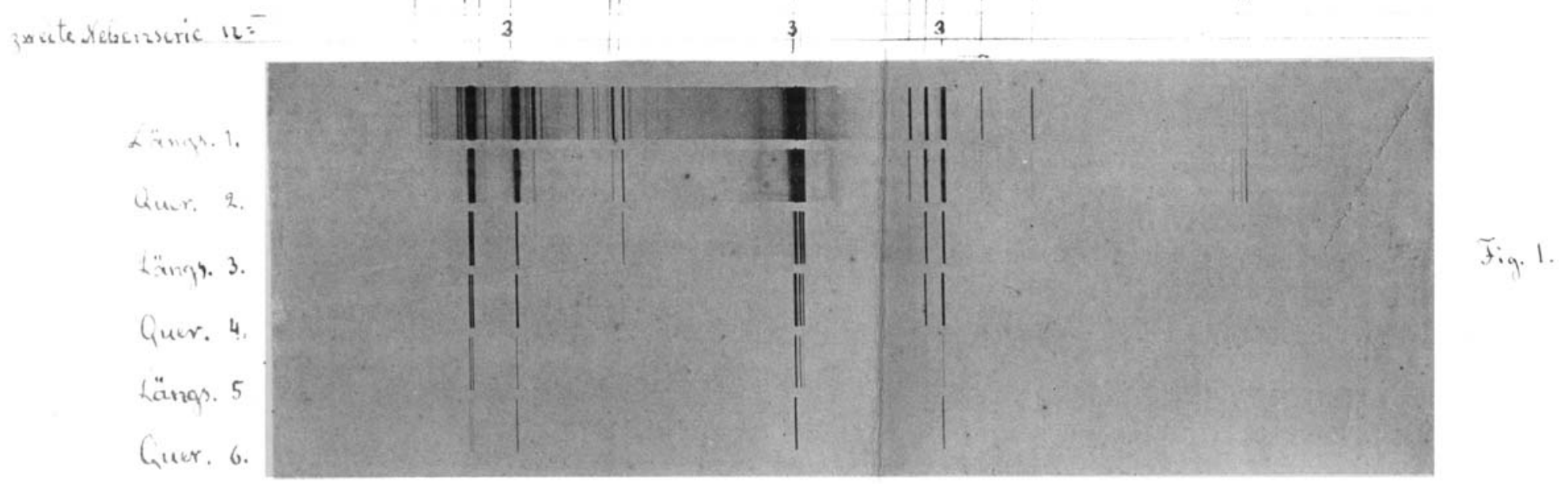

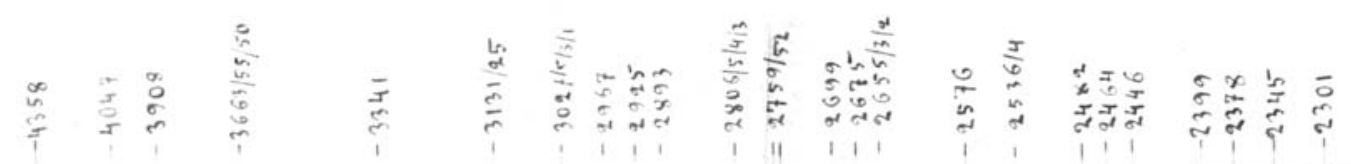

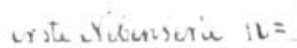
arciteriecheria $n=$

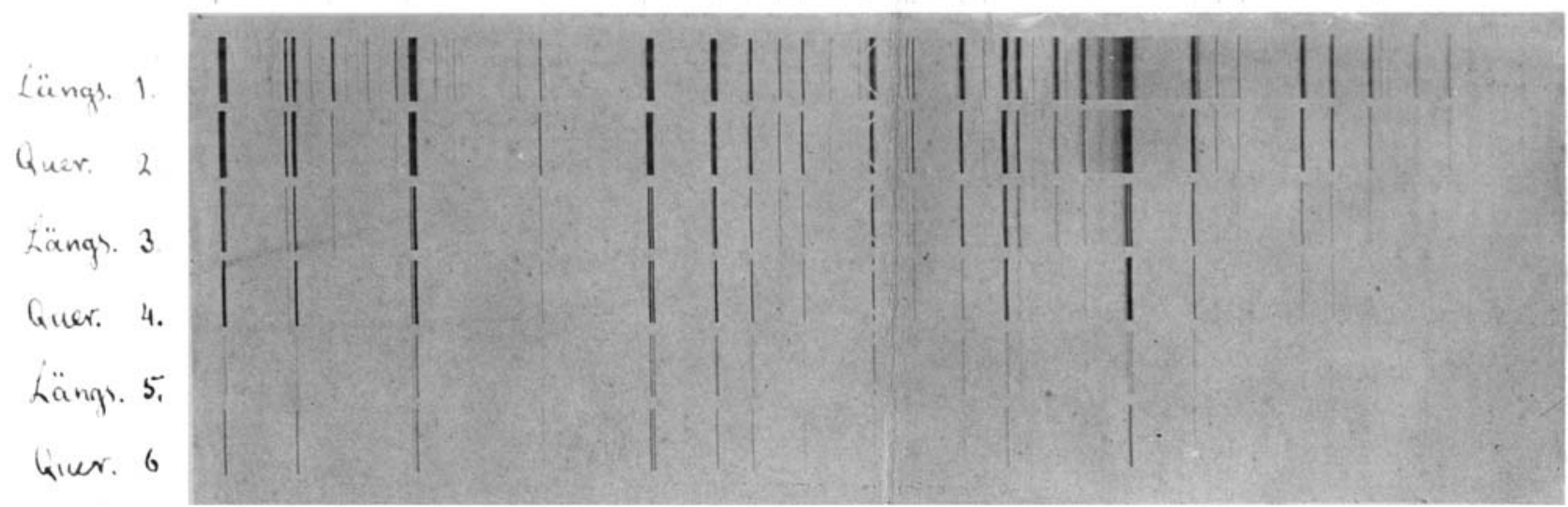

